\title{
The protective role of acupuncture after skin graft surgery
}

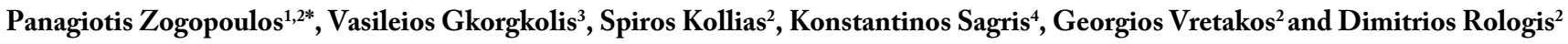 \\ ${ }^{1}$ Department of Medical Acupuncture, Metropolitan hospital, Athens, Greece \\ ${ }^{2}$ Department of Neurosurgery, Metropolitan hospital, Athens, Greece \\ ${ }^{3}$ St. Andrews Centre for Plastic Surgery and Burns, Broomfield Hospital, Chelmsford, UK \\ ${ }^{4}$ Intensive Care Unit, Metropolitan hospital, Athens, Greece
}

\begin{abstract}
Acupuncture has been widely used for many centuries in Asia but also, over the last decades, in Western countries, as a treatment modality for various diseases and disorders. Recent advances have shed light on the mechanisms of action of acupuncture treatment and have greatly expanded its potential indications. We present a mini-review of available experimental and clinical data, to date, regarding acupuncture's protective effects after skin graft/flap reconstructive surgery.
\end{abstract}

\section{Introduction}

Acupuncture is a treatment modality with a history of many centuries that originates from China. Over the last decades acupuncture has been incorporated into western medical practice and is thus, referred to as medical acupuncture. Medical acupuncture is under extensive investigation regarding its potential indications and underlying mechanisms of action. We present here current evidence, to date, regarding acupuncture's potential beneficial effects on skin graft/flap survival.

\section{Materials and methods}

We reviewed all available publications on medical database PubMed, regarding acupuncture and its effects on skin graft survival. Search terms were "acupuncture", "skin graft/flap" and "skin/cutaneous blood flow". In total, twelve studies were included [both clinical and experimental].

\section{Results}

\section{Experimental data}

Experimental animal studies have shown that acupuncture and its most potent form, electroacupuncture, can significantly increase survival of skin flaps/grafts compared to untreated controls [1]. Specifically, electroacupuncture treatment has been associated with more than $90 \%$ skin graft survival rates [1].

In an experimental animal [rat] study, McFarlane skin flaps were established, and subjects received electroacupuncture stimulation [2]. This led to a significantly larger mean survival area of skin flaps, compared to the control group [2]. Furthermore, immunohistochemical analysis showed that vascular endothelial growth factor (VEGF) expression level and superoxide dismutase activity was also significantly higher in the electroacupuncture group than in the control group [2]. Finally, skin flap tissues examined in hematoxylin and eosin-stained slices exhibited significantly less signs of inflammation compared to controls [2].

Blood reflow and reoxygenation of ischemic skin flaps may lead to tissue damage through free radical's production [3]. Acupuncture and electroacupuncture treatment led to a significant decrease of skin myeloperoxidase activity in skin grafts, as well as a decrease of plasma and skin glutathione levels in another experimental rat model [3]. This explains the reduced inflammation and oxidative stress observed in these specimens, compared to control group and these findings are consistent with acupuncture's well-established anti-oxidative properties in various organs and tissues [3].

Electroacupuncture treatment in an experimental rat model of skin grafts has been associated with a significantly higher skin flap survival index and lower rate of necrosis, compared to control group and suggested that electroacupuncture may be an efficient treatment modality to preserve skin graft vitality [4]. In another experimental rat model electroacupuncture led to increased blood flow at the periphery of musculocutaneous grafts, compared to controls [5]. Doppler flowmetry studies have found that electroacupuncture treatment increases cutaneous blood flow and consequently contributes to skin graft survival [6]. This beneficial effect on cutaneous blood flow was equivalent to injection of substance $\mathrm{P}$ or calcitonin gene-related peptide (CGRP) [6].

\section{Clinical data}

Clinical studies on healthy volunteers have revealed that acupuncture treatment can induce various physiological changes [7]. In particular, a significant increase in local skin temperature has been observed [7]. Acupuncture treatment has been found to induce a transient, early increase in skin sympathetic nerve activity, accompanied by an initial reduction and, thereafter, increase of skin blood flow [8]. These changes were induced by a segmental effect of spinal nerves on local cutaneous microcirculation [8-9]. Calcitoningene related peptide (CGRP), a potent endogenous vasodilator, seems to be playing a major role in this spinal nerve-induced skin blood flow alteration [9].

Correspondence to: Zogopoulos P, Department of Medical Acupuncture, Metropolitan hospital, Athens, Greece; E-mail: p.zogopoulos@yahoo.com

Received: January 01, 2018; Accepted: January 12, 2018; Published: January 15,2018 
A clinical study on healthy volunteers showed that, even 5 minutes of low frequency $(1 \mathrm{~Hz})$ electroacupuncture stimulation could increase both skin blood flow and muscle blood volume and this increase was statistically significant compared to the control group [10]. Laser Doppler perfusion imaging studies have shown that acupunctureinduced cutaneous vasodilation is mediated, at least in part, through upregulation of nitric oxide (NO), in addition to a possible axon reflex mechanism, since NO synthase inhibitor administration reversed the vasodilatory effects of acupuncture [11].

Another clinical study investigated the effects of acupuncture on skin and muscle blood flow, utilizing photoplethysmography [12]. Compared to control group, acupuncture arm exhibited pronounced increase of skin and muscle blood flow, but only after acupuncture with needle manipulation technique (the so-called DeQi stimulation) [12]. These results suggested that the effects of acupuncture on skin and muscle blood flow were largely dependent on the intensity of needling stimulation [12].

\section{Discussion}

Random skin grafts/flaps are commonly used for wound repair and reconstruction in plastic surgery. Tissue ischemia is, probably, the worst complication in skin graft/flap surgery and, when it occurs, can lead to widespread necrosis and skin graft rejection. Various factors are important in maintaining skin graft blood flow and, thus, survival including skin graft size, shape and thickness, local vasculature and surgical technique (e.g. suturing avoiding tension).

Skin blood flow has been found to be influenced by two specific mechanisms: a segmental nerve axon reflex and endothelial release of nitrix oxide (NO), which is a potent vasodilator [13]. The initially occuring axon reflex is believed to be mediated by the release of the neuropeptides substance $P$ and calcitonin gene-related peptide (CGRP) [14-15]. Nitric oxide is known to play a role in the release of CGRP in the skin and also contributes to substance P-mediated dilatation [14-15]. Since acupuncture has been found to upregulate NO in local circulation, its effect in the cascade that finally causes vasodilation and skin blood flow increase may be rather important [11].

\section{Conclusion}

Acupuncture treatment and its most potent form, electroacupuncture, can enhance local microcirculation and blood perfusion and thus, significantly increase skin graft/flap survival.
Furthermore, it can also be used as an effective treatment of local ischemia, would it occur after skin graft reconstructive surgery.

\section{References}

1. Jansen G, Lundeberg T, Samuelson UE, Thoma M (1989) Increased survival of ischaemic musculocutaneous flaps in rats after acupuncture. Acta Physiol Scand 135: 555-558. [Crossref]

2. Wang LR, Cai LY, Lin DS, Cao B, Li ZJ (2017) Effect of Electroacupuncture at The Zusanli Point (Stomach-36) on Dorsal Random Pattern Skin Flap Survival in a Rat Model. Dermatol Surg 43: 1213-1220. [Crossref]

3. Lima LP, de Oliveira Albuquerque A, de Lima Silva JJ, Medeiros Fd, de Vasconcelos PR, Guimarães SB (2012) Electroacupuncture attenuates oxidative stress in random skin flaps in rats. Aesthetic Plast Surg 36: 1230-1235. [Crossref]

4. Uema D, Orlandi D, Freitas RR, Rodgério T, Yamamura Y, et al (2008) Effect of eletroacupuncture on DU-14 (Dazhui), DU-2 (Yaoshu), and Liv-13 (Zhangmen) on the survival of Wistar rats' dorsal skin flaps. J Burn Care Res 29: 353-357. [Crossref]

5. Niina Y, Ikeda K, Iwa M, Sakita M (1997) Effects of electroacupuncture and transcutaneous electrical nerve stimulation on survival of musculocutaneous flap in rats. Am J Chin Med 25: 273-280. [Crossref]

6. Jansen G, Lundeberg T, Kjartansson J, Samuelson UE (1989) Acupuncture and sensory neuropeptides increase cutaneous blood flow in rats. Neurosci Lett 97: 305309. [Crossref]

7. Li W, Ahn A2 (2016) Effect of Acupuncture Manipulations at LI4 or LI11 on Blood Flow and Skin Temperature. J Acupunct Meridian Stud 9: 128-133. [Crossref]

8. Kimura K, Masuda K, Wakayama I (2006) Changes in skin blood flow and skin sympathetic nerve activity in response to manual acupuncture stimulation in humans. Am J Chin Med 34: 189-196. [Crossref]

9. Jan YM, Li TC, Hsieh CL (2010) A segmental effect involved in the changes of skin blood flow induced by acupuncture in normal health human. Am J Chin Med 38: 441448. [Crossref]

10. Mori H, Kuge H, Tanaka TH, Taniwaki E (2014) Influence of different durations of electroacupuncture stimulation on skin blood flow and muscle blood volume. Acupunct Med 32: 167-171. [Crossref]

11. Kimura K, Masuda K, Wakayama I (2006) Changes in skin blood flow and skin sympathetic nerve activity in response to manual acupuncture stimulation in humans. Am J Chin Med 34: 189-196. [Crossref]

12. Sandberg M, Lundeberg T, Lindberg LG, Gerdle B (2003) Effects of acupuncture on skin and muscle blood flow in healthy subjects. Eur J Appl Physiol 90: 114-119. [Crossref]

13. Cable NT (2006) Unlocking the secrets of skin blood flow. J Physiol 572: 613 [Crossref]

14. Minson CT, Berry LT, Joyner MJ (2001) Nitric oxide and neurally mediated regulation of skin blood flow during local heating. J Appl Physiol (1985) 91: 1619-1626. [Crossref]

15. Kellogg DL Jr, Liu Y, Kosiba IF, O’Donnell D (1999) Role of nitric oxide in the vascular effects of local warming of the skin in humans. J Appl Physiol (1985) 86 1185-1190. [Crossref]

Copyright: (C2018 Zogopoulos P.This is an open-access article distributed under the terms of the Creative Commons Attribution License, which permits unrestricted use, distribution, and reproduction in any medium, provided the original author and source are credited. 